Voix et Images

\title{
Paul-Marie Lapointe, le combinateur et le jazzman
}

\section{Robert Major}

Volume 6, numéro 3, printemps 1981

Philippe Haeck

URI : https://id.erudit.org/iderudit/200282ar

DOI : https://doi.org/10.7202/200282ar

Aller au sommaire du numéro

Éditeur(s)

Les Presses de l'Université du Québec

ISSN

0318-9201 (imprimé)

1705-933X (numérique)

Découvrir la revue

Citer cet article

Major, R. (1981). Paul-Marie Lapointe, le combinateur et le jazzman. Voix et Images, 6(3), 397-408. https://doi.org/10.7202/200282ar d'utilisation que vous pouvez consulter en ligne.

https://apropos.erudit.org/fr/usagers/politique-dutilisation/ 


\title{
Paul-Marie Lapointe, le combinateur et le jazzman
}

\author{
par Robert Major
}

Depuis sa publication en 1959 dans la revue Liberté. Arbres n'a cessé de fasciner. Le contexte socio-politique de l'heure explique en partie le premier émerveillement face à ce très beau poème, le plus connu de Lapointe. Au sortir de ce qu'il est convenu (mais pour combien longtemps encore?) d'appeler "la grande noirceur», lors des premières flexions qui précédèrent notre «grand bond en avant , il n'est guère surprenant que ce texte ait eu des résonances profondes:

C'était en la fatidique année 1960 et, à une jeunesse qui voyait s'ouvrir devant elle les portes de l'avenir, le poète offrait une image d'enracinement, de puissance et d'envol, une image du pays physique qui, sans sacrifier aux anciens mythes nationalistes et à la couleur locale, faisait du paysage le signe et le lieu d'une existence habitable.'

Dans un des meilleurs textes critiques qu'ait publié Partipris - Partipris qui incarne mieux que tout autre mouvement de l'époque, mieux que l'Hexagone, mieux que Liberté, mieux que l'Équipe du tonnerre, la volonté de prendre en main notre destin collectif - Paul Chamberland reconnaît justement en Arbres la pièce centrale de la thématique fondamentale de ses contemporains: la fondation et l'appartenance. ${ }^{2}$

Ainsi, à l'instar de quelques autres textes privilégiés - dont Mystère de la parole d'Anne Hébert, publié au même moment - Arbres prend valeur totémique: véritable objet qui synthétise la sensibilité et la problématique d'une époque, et par suite ses valeurs et ses aspirations.

Mais voilà: Arbres fascine encore. Presque vingt ans plus tard, alors que l'enthousiasme de l'intelligentsia québécois s'est sensiblement tempérée et que l'heure, sans être tout à fait au cynisme, est du moins à la sobriété et à la pondération teintées de la résignation qui vient de la conscience des limites (la politique avec ses compromissions et ses aléas ayant succédé à la pureté poétique - voir Gérald Godin - et les pôles mythiques de libération et de récupération du territoire que furent, au début des années soixante, les pays en lutte décolonisatrice, ayant décu - voir le Cuba, voir l'Algérie, voir la Guinée de Sekou Touré, ami de Frantz Fanon -). Fascination mystérieuse, venant en partie du fait qu'à la lecture du poème, il nous semble être en présence d'une 
des figures mythiques du poète, le poète inspiré qui, par le seul pouvoir des mots (et, dans ce cas-ci, des mots réduits à leur plus simple expression, puisque le poème atteint un degré d'agrammaticalisme quasi insurpassable), réussit à envoûter. Lapointe lui-même, dans un texte théorique d'une importance capitale, semble valider cette perception de sa fonction, lorsqu'il insiste sur la poésie comme improvisation, ad libitum, par opposition à la poésie artisanale:

La plus haute forme de poésie, comme la plus haute forme d'art, est l'improvisation, qui ne met aucun frein a l'expression, bien qu'elle tire son excellence d'un artisanat préalable, aussi bien sur la matière du créateur que sur celle du matériau.

La forme d'improvisation particulière au jazz - ad libitum sur une structure donnée, linéaire et verticale - me paraît devoir exprimer de la façon la plus concrète la forme de la nouvelle poésie.

Sur le plan formel (et cela est réalisé par après, et comme pour permettre de vérifier le jazz du poème) la reprise d'un thème sur différents modes crée l'identité. II peut s'agir d'un thème, le plus simple, mais comportant en lui-même une complexité autorisant la modulation, en lui-même c'est-à-dire dans le poète créateur. On retrouvera son chemin dans le poème, à travers ses différentes phases et même les plus audacieuses, les plus excentriques, soit les plus imagées et les plus lyriques, les plus éloignées du thème initial, par des mots ou des phrases, proférées ou tues, comme un navire naviguant entre des bouées, ou comme le jazziste se raccroche à la terre en repartant des mêmes séries d'accords ou d'une mélodie sous-jacente et constamment mère, pour créer son jazz et transmettre la parole, compte rendu, inventaire, colère ou prophétie.

Cette poésie diffère des poésies classiques, qui sont d'abord artisanales, ciselées, ouvrées au sens d'une quête de la perfection formelle. ${ }^{3}$

A vrai dire, l'antithèse poésie improvisée/poésie artisanale let, implicitement, poète inspiré/poète artisan) n'est guère absolue. L'improvisation «tire son excellence d'un artisanat préalable», comme le reconnaît lui-même Lapointe. Et le poète, comme le jazzman, est «l'homme d'un métier, qui connaît toutes les recettes de son art pour en avoir fait longuement l'apprentissage en se mettant à l'école des maîtres" 4 . C'est ainsi, selon Mikel Dufrenne, que composaient les prophètes hébreux, les aèdes grecs, les bardes celtes et les gondoliers vénitiens : le poète étant celui qui incarne le langage, qui, en bon ouvrier, s'est soumis à une longue discipline pour l'imprimer dans sa chair, l'improvisation lui est devenue possible; la mémoire du corps conduit la parole vers de nouvelles aventures. ${ }^{5}$

Poésie artisanale/poésie inspirée : pour qui connaît les sources d'Arbres, ce poème semble réaliser une véritable totalisation dialectique d'une double démarche apparemment antinomique : la reprise fidèle d'un texte préexistant, mais son éclatement constant par des images et des associations hardies, résolument personnelles. II emprunte le fonctionnement de l'ancienne rhétorique mais, simultanément, donne libre cours à une imagination proprement 
surréaliste. L'allure d'improvisation absolue et de foisonnement bienheureux, en somme de créativité sans brides, étant l'aspect que l'on retient le plus souvent à la lecture d'Arbres, je voudrais, quant à moi, insister surtout sur sa dimension de texte fabriqué.

Le commentator.

Le commentator s'introduit bien dans le texte recopié, mais seulement pour le rendre intelligible. ${ }^{6}$

\section{1) Contre une lecture nationaliste}

On sait généralement que Lapointe s'est inspiré d'un traité de botanique pour rédiger son poème: un dimanche, en plein cœur de Montréal, après avoir lu Arbres indigènes du Canada, il s'est installé à sa machine à écrire et a rédigé d'un trait son œuvre, qu'il retravaillera par la suite ${ }^{7}$. On ne peut guère deviner, toutefois, avant de s'être livré à une lecture méthodique et parallèle du poème et de ses sources, jusqu'à quel point Lapointe est fidèle à ces dernières. Cette fidélité influe nécessairement sur la signification du poème, du moins négativement. Elle empêche ou du moins invalide sérieusement certain type de lecture, trop étroitement nationaliste. II est révélateur, par exemple, que de préférence au frère Marie-Victorin, ce classique québécois dont la Flore laurentienne est une œuvre touffue et riche, pleine de détails pittoresques de tout ordre: folkloriques, historiques, économiques, culturels, ethnologiques, Lapointe ait privilégié une publication fédérale, plus sèche et pancanadienne: Arbres indigènes du Canada, de R.C. Hosie ${ }^{8}$.

Cette préférence entraîne un certain nombre de conséquences. A la suite de Hosie, le poète évoque un bon nombre d'espèces qui ne poussent pas au Québec. Dès le début de l'inventaire, avec le premier genre nommé (le pin), le lecteur informé est averti:

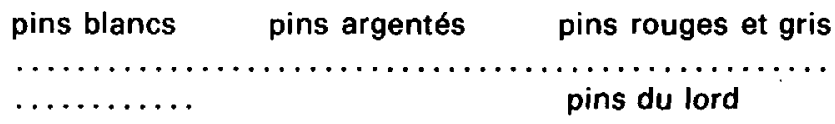

Le pin argenté est un autre nom du pin blanc de l'ouest (pinus monticola) qui ne pousse qu'en Colombie britannique; le pin du lord est sans doute (par association avec le premier gouverneur britannique du Canada), le pin de Murray, autre espèce de l'ouest, communément appelée pin lodgepole. Le même phénomène se répètera tout au long du poème : le cyprès jaune, le sapir concolore, le sapin gracieux, le sapin grandissime sont des espèces de la Colombie britannique; le peuplier aux feuilles étroites ne pousse qu'er Saskatchewan et en Alberta; l'aune crispé est très rare au Québec; le châtaignier ne vient que dans le sud de l'Ontario, de même que le chêne des marais et les fèvriers (chicot du Canada ou gymnoclade); le marronnier est importé d'Italie et quasi inexistant au Québec. 
Ainsi, tout en consultant Flore laurentienne (nous en verrons des indications ci-dessous), Lapointe préfère suivre Hosie. Un vers est surtout significatif à cet égard:

sureau bleu alouette sifflet dans les doigts

II s'agit de la dernière espèce nommée, juste avant la finale du poème. Les associations *alouette sifflet dans les doigts»s'expliquent par une indication du bon frère:

le nom générique [du sureau] dérive du nom grec d'une espèce de flûte (sambusque) que l'on fabriquait jadis avec le bois creux du sureau. (M.V., p. 530)

Toutefois, Lapointe passe sous silence les trois espèces de sureau nommées par Marie-Victorin (sureau yièble, sureau blanc, sureau rouge) et évoque la seule espèce nommée par Hosie, le sureau blanc, qui ne pousse qu'en Colombie Britannique!

Toute « explication n nationaliste du poème est donc au départ discutable. La démarche première du poète est certainement d'établir, en même temps que la présence de l'arbre, ses multiples relations avec l'homme, dans un mouvement réciproque (humanisation-arborisation) d'apprivoisement; mais faire d'Arbres le poème de la prise de possession du territoire, de l'enracinement de I'homme d'ici dans son sol (entendons: le Québec), est certainement réducteur ${ }^{9}$.

\section{2) Recopier}

Paul-Marie Lapointe semble s'être placé - à contre courant de la valorisation moderne de l'originalité et de la création - dans une relation essentiellement médiévale face à l'œuvre:

Ce que par anachronisme nous pourrions appeler l'écrivain est donc essentiellement au moyen âge: 1) un transmetteur: il reconduit une matière absolue qui est le trésor antique, source d'autorité; 2) un combinateur: il a le droit de 'casser' les œuvres passées, par une analyse sans frein, et de les recomposer (la 'création', valeur moderne, si l'on en avait eu l'idée au moyen âge, y aurait été désacralisée au profit de la structuration). ${ }^{10}$

Dans la série des fonctions qui entourent alors l'écrit (le scriptor, le compilator, le commentator, l'auctor), Lapointe parait sous les traits du commentator: il recopie la matière que lui ont transmis les botanistes, mais dans la seule visée de la rendre «intelligible».

La fidélité de la reconduction de Lapointe n'est guère discutable. II reprend la série des genres consignée dans Hosie (pin, cèdre, épinette, sapin ...) et des espèces au sein des genres (pins blancs, argentés, rouges, gris ...) en se permettant à peine quelques dérogations mineures à l'ordre du botaniste. Ces variantes par rapport à la source ne semblent obéir à aucune finalité. II n'apparaît guère significatif que le cèdre et le genévrier, par exemple, soient 
en deuxième et troisième place dans le poème, alors que chez Hosie ils viennent en cinquième et sixième place, après et non avant l'épinette et le sapin. On pourrait certes supposer que le poète fait d'abord intervenir les espèces domestiquées au sens fort (rattachées à la domus, la maison): après le pin des armoires, le cèdre des coffres. Mais alors il faudrait expliquer pourquoi l'érable, espèce domestiquée par excellence, devenant nourriture humaine, est en quasi finale du poème, comme il est à la fin de la série botanique.

Lapointe copie. Et le lecteur est tout surpris, véritablement émerveillé de la puissance proprement poétique de ces séries de noms. Cela tient à la variété et au nombre des espèces, véritable foisonnement, richesse naturelle renversante, surtout pour le citadin dénaturalisé pour qui tout arbre n'est qu'un arbre. Cela tient aussi à la beauté et à la saveur propre de ces noms qui puisent à ce que Ponge appelait, dans $L a$ Fabrique du pré, l'innocence lle manque d'imposture) de la nomination originelle. Lapointe tire ici profit du pittoresque et de l'expressivité de ce qu'à défaut d'un meilleur terme on pourrait appeler une poésie populaire. Une tradition séculaire, suppléée à l'occasion par l'effort classificateur des scientifiques, lui fournit ces noms qui, pour un lecteur profane, semblent traduire une vision fraîche de l'arbre ou tendre à son humanisation («charme bois dur bois de fer», «caryer à noix piquées», «érable à sucre»...), ou toutes ces épithètes, surtout de couleur, foncièrement ambiguës dans leur fonctionnement synecdochique (car enfin, qu'est-ce qui est blanc dans l'épinette blanche?) II s'agit d'une espèce de poésie des sources, la variété des épithètes tenant de l'arbitraire (il n'y a aucune différence de couleur entre l'épinette blanche et l'épinette noire, mais une différence de taille, de cônes, de feuilles), et étant imposée par l'usage (populaire ou scientifique), de sorte que le poète n'a rien à créer. Dans la très belle série:

sapins blancs sapins rouges concolores et gracieux
sapins grandissimes

par exemple, rien n'est inventé: les dernières épithètes, plus frappantes, sont celles mêmes qui désignent les espèces, et sous leur nom scientifique (Abies concolor, abies amabilis, abies grandis: Hosie, p. $86,92,94)$ et sous leur nom français.

Répétitions anaphoriques (sapins...sapins...sapins...), multiplication des synecdoques (chacune des déterminations de couleur et plusieurs des noms - par exemple, le merisier - sont soit synecdochiques, soit purement arbitraires), foisonnement des épithètes avec ce que cela implique de redondance rhétorique (que l'on se rappelle que Fontanier range l'épithète parmi les figures du discours ${ }^{11}$ ), déterminations insolites (surtout sous forme de compléments de nom: le cèdre de l'est est de fait le thuja occidentalis...): la "source d'autorité" qu'exploite Lapointe fourmille de procédés poétiques. Et cela, indépendamment des arbres eux-mêmes qui sont des objets poétiques (qu'on le veuille ou non, comme la lune, dont Mallarmé devait bien, à son corps défendant, admettre: “elle l'est, la garce»), Le traité de botanique lui offrait sur un plateau d'argent, surtout dans la richesse des déterminations spécifiques, un matériau d'élection. 
Par ailleurs, Hosie et Marie-Victorin, malgré l'indéniable intérêt de leurs propos, n'ont pas fait de la poésie. Quelle transformation leur a fait subir Paul-Marie Lapointe pour justement composer un très beau poème?

\section{3) Rendre «intelligible»}

Reprenons la merveilleuse définition de Barthes dans son sens le plus large. II est certain que Lapointe "s'introduit» dans le texte recopié et qu'à tout moment il le fait éclater. Le rend-il plus intelligible? Si, à condition d'entendre par «intelligible» non pas une certaine accessibilité réservée à l'entendement (les photos, les croquis, les descriptions détaillées et la taxinomie des botanistes ont une indéniable supériorité sur ce plan), mais plutôt une nouvelle clarté, toute particulière, appartenant en propre à la poésie. La poésie que Maritain appelait "cette intercommunication entre l'être intérieur des choses et l'être intérieur du Soi humain qui est une sorte de divination (comme l'Antiquité le savait bien; le vates latin était à la fois un poète et un devin)» 12 . Les arbres, sous la plume de Lapointe, acquièrent une présence, une épaisseur, une immédiateté, en somme, une intelligibilité qu'occultait l'«objective» présen.ation des botanistes (car lequel, du poème ou du traité, est davantage «objectif», fait davantage sentir l'objet?)

La disposition graphique joue un rôle privilégié à cet égard. C'est avec raison que Jean Cohen fait de la composition typographique le premier élément différenciateur et constitutif de la poésie, que celle-ci obéisse aux règles traditionnelles de la versification ou non ${ }^{13}$. Le blanc qui isole chacune des espèces, chacune des périphrases, chacune des perceptions imagées dans un monde de silence contribue fortement à conférer une valeur absolue aux arbres; le signe graphique de la pause devient en quelque sorte structuralement homologue au vide spatial qui isole chaque arbre dans l'immensité de la nature et le constitue dans sa présence concrète. Ces pauses sont à la fois métriques et sémantiques. Elles imposent un rythme morcelé et saccadé à l'élocution du poème, le rythme même de l'énumération, de l'inventaire, ou encore, de la litanie. Comme l'inventaire, Arbres a un caractère d'énonciation objective; comme la litanie, il est quelque peu nomination liturgique, prière invocatoire d'une extrême simplicité formelle au rythme envoûtant. Les «catholiques sociologiques» que nous sommes (athées et agnostiques de fraîche date compris: il paraît que le catholicisme ne lâche jamais son homme) ne peuvent rester insensibles au climat quasi révérentiel instauré par ce rythme monotone. II produit un sentiment presque panthéiste devant une nature d'une telle fécondité; près de cent espèces sont nommées, mais la série pourrait continuer car justement le propre d'une telle structure et d'un tel rythme est de pouvoir se prolonger indéfiniment : la conclusion intervient arbitrairement et $n$ 'implique aucunement un tarissement des forces vitales de la nature, ou des limites à la grandeur divine.

Ces pauses sont aussi sémantiques: en l'absence de toute ponctuation elles signalent «une articulation qui est à la fois psychologique et grammaticale "14. Elles sont le seul lien syntaxique de substantifs absolus privés de 
tout article et de tout verbe. Privés d'article, donc ni définis, ni indéfinis, ni parties d'un ensemble, mais purs; privés de verbes, dont le «propre est d'être sous-tendu de temps» 15 , donc intemporels, ces substantifs sont unis par le degré zéro de l'articulation syntaxique: la simple juxtaposition indifférenciée. Tout le poème se présente donc comme une mise en apposition sérielle à l'énoncé initial: «j'écris arbre» qui devient ainsi le "point de départ d'une dissémination libre, [donnée] comme naturelle " ${ }^{16}$. Le poème a donc lui-même une allure végétale, drageonnant follement. Lapinte exploite non seulement, dans la forme de son poème, cet élan vital particulier au règne végétal, il lui conjugue le foisonnement tout aussi remarquable de la langue. Car si la nature ne semble guère économe en multipliant les jeunes pousses, que dire de la langue qui, par surenchère, semble-t-il, rivalise de prolixité et désigne souvent la même essence de plusieurs façons différentes, semant les noms à la volée. Hosie en donne comme exemple dans son introduction, le merisier qui «se nomme également Bouleau jaune, Bouleau frisé, Bouleau dur, Bouleau noir. Bouleau doré, Bouleau argenté, Grand Bouleau et Bouleau rouge, à divers endroits de l'est du Canada» (p. 23). Cette redondance particulière aux noms vernaculaires sera utilisée systématiquement par les mises en apposition répétées, isolées par les blancs. Un seul exemple illustrera le procédé:

pins blancs pins argentés pins rouges et gris
pins durs à bois lourd pins à feuilles tordues

potirons et baliveaux

pins résineux

Le pin rouge est aussi le pin résineux (Hosie, p. 48). Le pin dur à bois lourd est aussi le pin à feuilles tordues; Hosie (pp. 46-47) présente le pin des corbeaux (pinus rigida) comme le «pin dur", décrit son bois comme étant «passablement lourd, relativement dur", et nous invite, sous une photo d'aiguilles, à «noter la torsion en spirale» (de là : «pins à feuilles tordues»). Mais le bois de ce pin des corbeaux est aussi «résineux», rejoignant ainsi, par le biais de son bois, le pin résineux, dont paradoxalement le bois n'est pas décrit comme étant " résineux". Il s'établit ainsi tout un jeu de correspondances et de renvois d'une grande complexité: «pins rouges» et «pins résineux» d'une part, «pins durs à bois lourd " et «pins à feuilles tordues» d'autre part, sont des noms différents d'une même espèce; mais «pins résineux» établit une relation surspécifique en étant d'une part le nom d'une espèce et d'autre part une tournure descriptive valable pour une autre. Ce qu'il faut surtout retenir, toutefois, c'est que ces déterminations (épithètes: blanc, rouge, gris; ou compléments de noms: à bois lourd, à feuilles tordues) fonctionnent ici à l'encontre du code normal. II ne s'agit pas, le plus souvent, de tournures descriptives mais de déterminations essentielles qui marquent l'espèce: la détermination est ici nomination. De la même façon que dire «un bel homme» c'est décrire tel homme, mais dire «un homme noir» c'est définir essentiellement tel autre dans ce que Sartre appelait un mouvement de «syncrétisme psycho-biologique » (cf. Orphée noir).

Ainsi, par la seule ressource de la composition typographique, d'une incroyable richesse sous son apparente simplicité (et dire qu'il s'en trouve 
encore pour croire qu'il s'agit de coquetteries de poète), Lapointe fait éclater le texte des botanistes, lui confere un relief extraordinaire. Les substantifs deviennent davantage substance, sous sa plume.

Cette sourde et sournoise transfiguration du texte en prose est encore plus évidente lorsqu'on examine de plus près le fonctionnement d'un certain nombre de périphrases dont le contenu est emprunté aux botanistes. Quelques exemples d'abord:

\section{Botanistes}

le pin gris «en sols pauvres et en terrain rocaileux... est court et souvent tordu» (Hosie, p. 50)

«le bois du pin rouge... est dur, léger et de longue durée, d'un usage général comme bois de mâture (M.V., p. 141)

le bois du cèdre rouge (genévrier de Virginie) « est utilisé notamment... pour la fabrication des crayons * (Hosie, p. 106)

la feuille du bouleau gris a de «petites dents s'intercalant entre les grandes» (Hosie, p. 166)

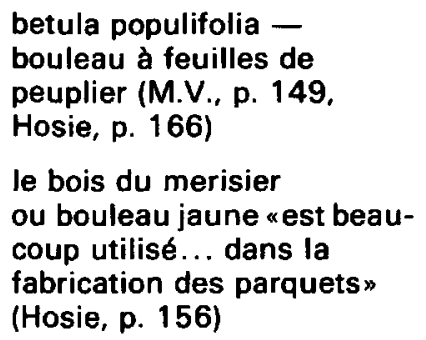

\section{Lapointe}

pins...chétifs et des rochers

pins...traversent les années mats fiers voiles tendues

genévrier qui tient le plomb des alphabets

feuilles-engrenage vidé

bouleau cambrioleur à feuilles de peuplier

bouleau des parquets

peuplier faux-tremble trembleur à grands crocs peuplier-loup griffon troubleur

saule écorce amère 
«les rameaux, généralement minces et flexibles, deviennent souvent cassants" (Hosie, p. 114)

«graines minuscules couvertes d'une touffe de longs poils blancs et soyeux» (Hosie, p. 114)

"les cicatrices foliaires sont surélevées sur des bourrelets du rameau * (Hosie, p. 168)

carya ovata - caryer à noix douces - arbre à noix piquées

(Hosie, p. 140)

«le bois est recherché pour la fabrication d'articles de sport» (Hosie, p. 140)

le bois du chêne blanc "est utilisé en ébénisterie, parqueterie, boiserie intérieure, construction de navires, tonnellerie (vins et autres liquides)"

(Hosie, p. 182)

cerisier d'automne (Hosie, p. 240)

bois barré - bois d'orignal: "une essence de sous étage"; "les feuilles et les jeunes pousses constituent une nourriture favorite des orignaux et des chevreuils, d'où le nom secondaire Bois d'orignal». (Hosie, p. 278) saule aux rameaux grêles cassants comme parole en l'air

graine-coq à aigrette et paon fugace

aunes à bourrelets

caryer à noix piquées au vif

caryer sportif

chêne...constructeur transport de soif

cerisiers d'octobre à l'année longue

érable barré bois d'orignal nourriture d'été fidèle au gibier traqué dans les murs et la fougère

Cette mise en parallèle n'a rien d'exhaustif. Les évocations facilement repérables, faisant partie du savoir commun («pins des calmes armoires», «cèdres bardeaux parfumeurs coffres des fiançailles", «épinette breuvage d'été»...) ont été évitées, et une sélection a été faite parmi les très nombreux emprunts de Lapointe à un savoir plus précis, celui des botanistes et des forestiers.

La disposition sur deux colonnes permet de voir assez facilement la liquidation de la grammaire qu'effectue Lapointe et la restructuration compressive à laquelle il se livre. La syntaxe est abandonnée au profit de la parataxe: le lien syntaxique disparaît complètement dans la mise en apposition ("saule 
écorce amère»), ou bien il est réduit à sa plus simple expression par la détermination élémentaire du complément de nom, sans procès ou mouvement aucun. Ces fusions radicales dégagent - comme toute fusion d'éléments normalement irréductibles, dont la relation est fortement médiatisée - de grandes quantités d'énergie: comme si nous étions en présence d'une série d'explosions dont l'irradiation aveuglait autant qu'elle éclairait. Un tel fonctionnement semble épouser étroitement la conception heideggerienne du langage poétique axée sur la puissance du mot: «la libération du langage des liens de la grammaire, en une articulation plus originelle de ses éléments, est réservée à la pensée et à la poésie»17. C'est ainsi, selon le philosophe, que la pensée et la poésie, libérées du langage-système, saisissent le réel de la façon la plus vive et la plus efficace possible.

Une lecture même rapide des quelques exemples donnés permet de voir au moins un quadruple aspect à cette saisie du réel dans Arbres. En premier lieu, des raccourcis qui fixent les arbres dans des images fulgurantes («pins ... chétifs et des rochers", "saule aux rameaux grêles cassants»); ensuite des juxtapositions qui construisent un véritable bestiaire des arbres («peuplier-loup griffon troubleur», «graine-coq à aigrette et paon fugace»); troisièmement, un début d'humanisation de l'arbre par sa transformation en objets utiles («bouleau des parquets», "mats fiers voiles tendues», «transport de soif»); enfin, une personnification totale de l'arbre («bouleau cambrioleur», «constructeur», "caryer sportif», "piquées à vif»). Cette classification n'est guère rigoureuse car il y a chevauchement constant des niveaux dans un jeu quasi euphorique du langage: l'aune "à bourrelets" est à la fois une image justifiée scientifiquement et une personnification heureuse (le «bourrelet», pris absolument, désignant un pli de chair sur le corps humain): "gibier entre I'homme et la bête, double gibier sauvé par l'arbre ; "genévrier qui tient le plomb des alphabets" décrit l'objet, le crayon, mais dans un mouvement de personnification. Les nombreux jeux de mots créent de plus une dimension ludique appréciable: c'est ainsi que le cerisier d'automne devient «cerisiers d'octobre à l'année longue» et le tremble un "trembleur", un peureux malgré son aspect redoutable. Par le jeu du langage, s'instaure un tissu complexe de relations ambivalentes (arbre-monde ambiant, arbre-animal, arbre-objet, arbre-homme) qui glissent rapidement dans la plurivalence, chacun des éléments entretenant des liens multiples avec tous les autres.

Quand Lapointe délaissera le texte des botanistes pour se livrer à de véritables mouvements d'improvisation, il ne procèdera pas autrement: images saisissantes, bestialisation, humanisation, personnification:

conifères d'abondance
vertes des matinaux espèces hérissées crêtes
nifères cophandriers du vent dons quichottes sans monture sinon la
montagne
conifères clairons droits foudroyant le ciel
gelées de feu flammes pétrifiées vertes brûlantes
arêtes de poissons verticaux dévorés par l'oiseau


A cette différence (capitale) près, toutefois, que les métamorphoses de l'arbre pourront aussi devenir imprévues, déroutantes, cherchant à englober l'ensemble de la réalité (et de la surréalité). Se dégage alors, parmi d'autres, la signification suivante: puisque tout est signe de l'arbre, l'arbre est totalité dialectique, intégrant même la réalité la plus apparemment réfractaire à l'ordre naturel:

l'arbre est clou et croix croix de rail et de papier croix de construction croix de bombardier $\begin{array}{cc}\text { d'épée } & \text { de fusil } \\ \text { téléphone } & \text { haut fourneau }\end{array}$ sémaphore croix d'aluminium et de néon croix de gratte-ciel et de chien de torture et de faim

et le poème est essentiellement entreprise totalisante, foyer de convergence du réel.

Dans cette visée prométhéenne, le Lapointe combinateur qui, par le jeu de la graphie et l'exploitation radicale de la dislocation syntaxique, déstructure un texte initial pour lui conférer d'autres valeurs et d'autres sens, sera admirablement relayé par le jazzman. l'improvisateur de génie.

1. Gilles Marcotte, Le temps des poètes. Description critique de la poésie actuelle au Canada français, Montréal, HMH, 1969, pp. 71-72.

2. Paul Chamberland, Fondation du territoire, dans Parti pris, vol. IV, nos 9-10-11-12, mai-août 1967, p. 35.

3. Paul-Marie Lapointe, Notes pour une poétique contemporaine, dans Guy Robert, éd., Littérature du Québec, t. I, (Témoignages de 17 poètes), Montréal, Déom, 1964, p. 88.

4. Mikel Dufrenne, Le poétique, Paris, Presses Universitaires de France, 1963, p. 93.

5. Ibid., p. 94.

6. Roland Barthes, L'ancienne rhétorique, dans Communications, 16, 1970, p. 185. Souligné dans le texte.

7. Gaétan Dostie, Paul-Marie Lapointe, The Seismograph of Quebec, dans Ellipse, no 11, 1972, p. 61.

8. R. C. Hosie, Arbres indigènes du Canada, Ottawa, Ministère de l'Environnement, 1972, 383 p. (Dorénavant Hosie dans le texte).

Frère Marie-Victorin, Flore laurentienne, Montréal, Imprimerie de La Salle, 1935. 917 p. (Dorénavant M.V. dans le texte).

9. Ainsi Chamberland: $\alpha_{\ldots} .$. L'appropriation du territoire est soigneusement effectuée : la diversité est celle d'un inventaire du pays, du lieu anatal , où l'arbre est, à la faveur d'un prodigieux survol métonymique, investi de la fonction de « représenter o le territoire en ses aspects multiples. Notons que la multiplicité renvoie ici à la détermination du singulier, au spécifique». (Op. cit., p. 37). Ce «spécifique» est ambigu, si l'on peut s'exprimer ainsi : on ne saurait affirmer qu'il s'agit du seul Québec. Et même, petit détail sans conséquences mais ironique, puisque Lapointe copie aussi les erreurs de Hosie, il évoque une espèce propre aux U.S.A. (cf. Hosie, p. 90: $\propto$ Dans les éditions précédentes de ce livre, cette espèce était appelée $\alpha$ Sapin concolore $\%$. Le véritable Sapin concolore (Abies concolore), nommé communément Sapin du Colorado, n'est pas indigène au Canada».) Ce «spécifique» manque décidément de spécificité.

10. R. Barthes, op. cit., p. 185 . Souligné dans le texte. 
11. Pierre Fontanier, Les figures du discours, Paris, Flammarion, (Coll. Champs), 1977, p. 324.

12. Jacques Maritain, L'intuition créatrice dans l'art et dans la poésie, Paris, Desclée de Brouwer, 1966, p. 1. Souligné dans le texte.

13. Jean Cohen, Structure du langage poétique, Paris, Flammarion, (Coll. Champs), 1966 , p. 55 ss.

14. Ibid., p. 57.

15. G. Guillaume, cité par Le Petit Robert, article «verbe».

16. Pierre Nepveu, Les mots à l'écoute. Poésie et silence chez Fernand Ouellette. Gaston Miron et Paul-Marie Lapointe, Les Presses de I'Université Laval, 1979.

17. Martin Heidegger, Questions III, Paris, Gallimard, 1966, p. 75. 